one pioneer superintendent mourned in 1909: "Society only desires to get rid of [the mentally retarded] and be protected from them [whereas] the older ideas were to uplift them by every means that could be used. Now when thus congregated in Droves like cattle it is about as much as we can accomplish to keep them comfortable and fed and clothed."

The 'feebleminded' thus became 'mental defectives', and with a new implication: that their restraints on behaviour as well as their mental processes were blighted. They were the 'defective delinquents' who threatened social stability and prosperity. Because mental deficiency could be viewed as a simple Mendelian trait, the complementary strategies of segregation and eugenic sterilization were introduced. US communities also instituted specialeducation classes and parole as it became clear that there were too many defectives for the institutionalization of all those unable to 'adapt'.

After the 1930s, institutions became discredited following underfunding and scandals, and new technologies (especial- ly psychoactive drugs) began to lead to deinstitutionalization. Moreover, new advocacy groups, especially those involving family members, and university researchers began to replace superintendents as authorities on mental retardation. Reformers urged that rather than institutionalizing the retarded, normal community institutions should adapt to them ('mainstreaming'). Trent concludes that retarded people are not vegetables: many "hold full-time jobs, have families, and pay taxes - and wreck cars, have extramarital affairs, and get audited by the IRS [Internal Revenue Service]."

It is striking that, generations ago, the most severely mentally retarded were unable to cope with life in a producer culture, but in a consumer culture they have come to be consumers with the best of us - another redefinition of their and our - essential humanity.

John C. Burnham is in the Departments of History and of Psychiatry, Ohio State University. 230 West 17 th Avenue, Columbus, Ohio 43210, USA.

\title{
Dinosaur growth industry
}

\section{Angela Milner}

Dinosaur Eggs and Babies. Edited by Kenneth Carpenter, Karl F. Hirsch and John R. Horner. Cambridge University Press: 1994. Pp. 372. £55, \$79.95.

Do not be misled by the title, or by the cover illustration of a cute, gangling. down-clad titanosaurid hatchling. This is not a book aimed at the popular market but a collection of papers on all aspects of dinosaur reproduction and development for the professional reader and advanced student.

John Horner's discoveries of dinosaur nesting sites in the late Cretaceous strata of Montana, United States, during the late 1970s was the stimulus for a whole new slant on dinosaur research. Much of what is understood about dinosaur behaviour, reproduction and physiology is based on the study of nests, eggs, the growth and development of embryonic and juvenile skeletons, and the palaeoecology and environment of nest sites. This long-awaited book sets out to review and summarize today's knowledge of one of the most exciting areas of dinosaur studies.

The book consists of 24 separately authored chapters on distribution and history, nests, eggs and 'dinosaur babies'. The first chapter, a list of the global distribution of eggs, nests, bones, teeth and footprints, in chronological order, is a reference work in its own right.

Much of the information published here has been previously only tantalizingly hinted at in abstracts or referred to in passing as work in progress, and the lack of hard data has been the cause of some scepticism among vertebrate palaeontologists. A case in point is altricial (young relatively undeveloped when born hatched) and precocial (young at relatively advanced stage of development when born hatched) breeding patterns. New evidence for both patterns appears in papers in the 'babies' section, lending support to an idea suggested by Horner and David Weishampel in 1988 that altriciality, found so far only in large derived eusonithopods, may be a function of adult body size in that group.

Dinosaur growth rate is another controversial topic where debate has outstripped published data. In the book there is much discussion and general agreement, on the basis of histological and ontogenetic observations, that young dinosaurs grew rapidly. A novel approach by Weishampel and Horner addresses the importance of life-history syndromes and heterochrony in understanding dinosaur evolution and the authors attempt to analyse key shifts in the framework of a cladistic phylogeny while acknowledging that the database is currently sparse indeea. Descriptive contributions on embryonic and juvenile dinosaurs illustrate criteria by which such material can be recognized. This may sound obvious enough but, until recently, many workers failed to realize that smallness may indicate juvenility rather than a new taxon.
Study of dinosaur eggs and eggshell structure has evolved into a discipline of its own with a taxonomy of egg families, genera and species. Most of the recent studies have adopted a common system pioneered by the Chinese worker Z. K. Zhao in 1975. Several chapters demonstrate its value in analysing associations of eggs types and in interpreting nestingbehaviour patterns. So links and correlations can be made between taxonomic groups and environments in which the eggs were laid, for example between the rich Upper Cretaceous egg horizons in the Gobi Desert, central China and France even though the identity of the egg-layers is uncertain.

Eggshell studies also yield information about chemical content, pathology and physiology, including gaseous exchange, type of nest and position of eggs in it. Several chapters review these topics and two authors draw attention to the importance of changes that can alter shell structure during lithification of a sediment; recrystallization of eggshell calcium carbonate is common.

The bulk of evidence on dinosaur reproduction comes from Cretaceous strata, but rare records from the Triassic of Argentina and early Jurassic of South Africa suggest that nest contruction may have been plesiomorphic (that is, a hereditary character common to all dinosaurs). Egg-laying patterns are reviewed and two basic types, clutched (nest) and linear, are defined, with many variations of each type; a summary shows the extent to which the different types can be identified with dinosaur groups.

This work is an essential addition to the dinosaur literature and is the only compact reference work covering reproductive biology. It succeeds admirably in summarizing the current state of knowledge while drawing attention to how much remains unknown about dinosaur life histories. Tyrannosaurus rex's is still a complete mystery.

Angela Milner is in the Department of Palaeontology, Natural History Museum. Cromwell Road, London SW7 5BD, UK.

- Solnhofen: A Study in Mesozoic Palaeontologyby K. W. Barthel, N. H. M. Swinburne and S. Conway Morris has just been published in paperback by Cambridge University Press ( $£ 16.95, \$ 24.95$ ). One of the most famous limestones in the geological record, Solnhofen in southern Germany contains an astonishing diversity of organisms buried 150 million years ago, many of them exquisitely preserved: highlights include jellyfish, crustaceans, squid, fish, flying reptiles and - perhaps most famously - Archaeopteryx. "An excellent general survey . . . of the considerable body of research by German sedimentologists and taphonomists during the last 25 years" (Nature 249, 114; 1991). 